\title{
Institutional prevalence of malignancy of indeterminate thyroid cytology is necessary but insufficient to accurately interpret molecular marker tests
}

\author{
Pablo Valderrabano1, Marino E Leon², Barbara A Centeno², Kristen J Otto', \\ Laila Khazai ${ }^{2}$, Judith C McCaffrey ${ }^{1}$, Jeffery S RusselI ${ }^{1}$ and Bryan Mclver ${ }^{\mathbf{1}}$ \\ 'Department of Head and Neck, and Endocrine Oncology and '2Department of Anatomic Pathology, \\ H. Lee Moffitt Cancer Center and Research Institute, Tampa, Florida, USA
}

Correspondence
should be addressed
to B Mclver
Email
Bryan.mciver@moffitt.org

\begin{abstract}
Objective: Several molecular marker tests are available to refine the diagnosis of thyroid nodules. Knowing the true prevalence of malignancy (PoM) within each cytological category is considered necessary to select the most appropriate test and to interpret results accurately. We describe our institutional PoM among cytological categories and report our experience with molecular markers.

Design: Single-center retrospective study.

Methods: We calculated the institutional PoM for each category of the Bethesda system (Bethesda) on all thyroid nodules with cytological evaluation from October 2008 to May 2014. We estimated the predictive values for Afirma, miRInform, and ThyroSeq v2, based on published sensitivity and specificity. Finally, we assessed our own experience with miRInform.

Results: The PoMs for Bethesda III and IV categories were 21 and 28\%, respectively. ThyroSeq v2 achieves the highest theoretical negative and positive predictive values (NPV and PPV) in Bethesda III (98 and 75\%) and Bethesda IV categories (96 and $83 \%$ ). At our institution, miRInform detected a mutation in $16 \%$ of 109 indeterminate nodules tested, all in Bethesda IV specimens. Histology was available in 56 (51\%) nodules. The observed sensitivity and specificity in Bethesda IV specimens were 63 and $86 \%$, yielding an NPV and a PPV of 75 and $77 \%$, respectively. Conclusions: For our current Bethesda III and IV PoM, the actual performance of miRInform was worse than expected. Theoretically ThyroSeq v2 should have the best performance, but it could be affected in the same way as miRInform, given the similarities between the tests. Assessing the institutional performance of each test is necessary along with PoM individualization.
\end{abstract}

\section{Introduction}

Thyroid nodules with indeterminate cytology frequently undergo diagnostic surgery, which confirms malignancy in approximately one-third of cases. For these individuals, a second surgical procedure is often necessary to complete total thyroidectomy $(1,2)$; whereas for the other twothirds, surgery might have been unnecessary exposing the patient to the risk of complications (3). The rapid increase in the number of thyroid biopsies being performed in the
두 2016 European Society of Endocrinology Printed in Great Britain
European Journal of Endocrinology (2016) 174, 621-629
United States (seven-fold in the last decade) (4) has led to a parallel dramatic increase in the number of thyroid nodules with indeterminate cytology (1). In this context, several commercial tests involving the use of molecular markers have been developed over the past several years in an effort to refine the diagnosis of these nodules preoperatively. The long-term clinical and economic implications of their implementation in routine clinical

Published by Bioscientifica Ltd. 
practice are unknown. However, these tests are already in widespread use in the United States and have established a place in the recently revised version of the American Thyroid Association (ATA) guidelines and in the National Comprehensive Cancer Network (NCCN) Guidelines released in the past several years $(5,6)$.

The clinical utility of these tests relies on the negative and positive predictive values (NPV and PPV) attained, which are determined both by the intrinsic sensitivity and specificity of the test, and by the prevalence of malignancy (PoM) within the tested indeterminate categories. As the actual PoM for the indeterminate categories defined in the Bethesda system for reporting thyroid cytopathology (Bethesda) is highly variable between institutions, the performance of molecular markers tests needs to be individualized $(2,7)$. Because molecular marker tests are already integrated in our institution's thyroid nodule clinical pathway, we conducted this retrospective study to analyze and optimize their use at our academic cancer center, following the recommendations of the ATA. We also describe our initial experience with the test marketed as miRInform by Asuragen Inc. (Austin, TX, USA).

\section{Subjects and methods}

In this retrospective, institutional review board-approved study, we reviewed all the thyroid cytology evaluated at our institution from October 2008 to May 2014. Cytology reports in which the biopsy was performed on thyroid tissue remnants after thyroidectomy or in an organ other than the thyroid gland (lymph node, parathyroid gland, or other structure) were excluded (Fig. 1). Cytology results at our institution have been classified according to the Bethesda system since October 2008. In nodules with multiple biopsies, only the most recent cytology report was used for the study. Final histological diagnosis was obtained from review of histology reports. Only the histology of the biopsied nodule was used to assess the PoM in each Bethesda category; other nodules within the thyroid (including incidental malignancies in nodules that had not been biopsied) were not included in the denominator or the numerator of the calculation. In four nodules included in the study, focal areas of microcarcinoma were detected within an otherwise benign appearing nodule. These nodules were included in the malignant group, but the pathological size of the malignant foci was used as the pathological size of the tumor. Because the true PoM of each Bethesda category cannot be determined unless all thyroid nodules with

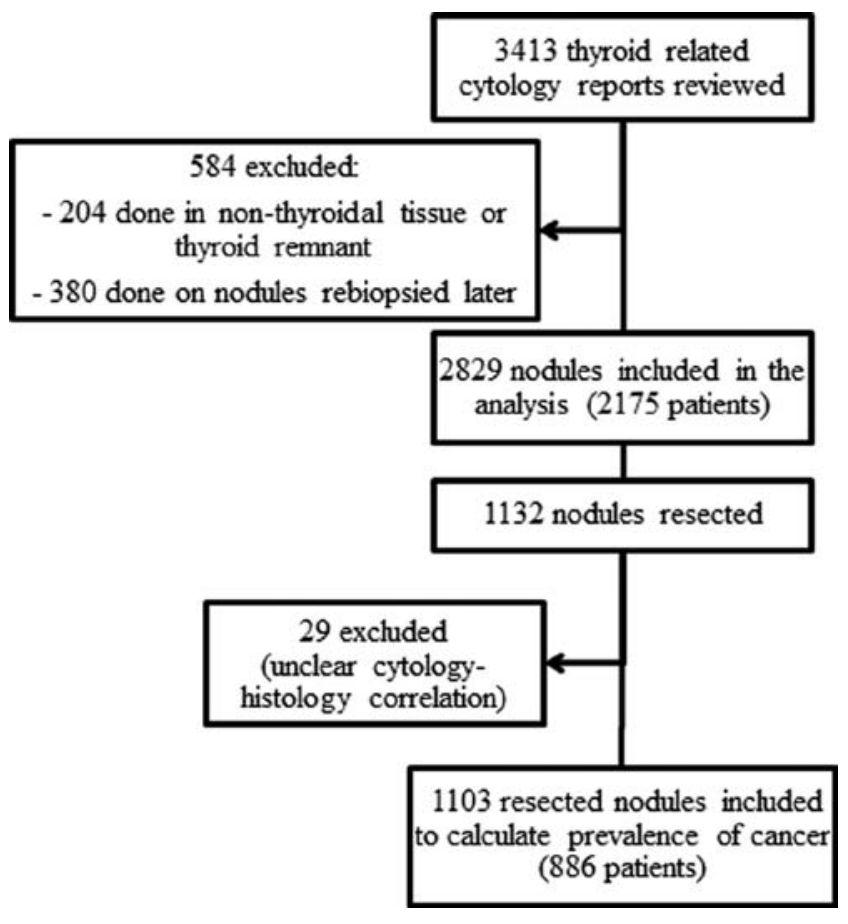

\section{Figure 1}

Study cohort selection process.

indeterminate cytology are resected, which is not clinically indicated in many cases, we calculated the bounds within which the 'true' PoM must lie for each group using three different approaches: First, we calculated the higher limit of our institutional PoM using only resected nodules for which there was a clear histological correlation. Because nodules with a cytologic diagnosis other than malignant may be more likely resected if they exhibit other high risk features, this approach may falsely raise the calculated PoM. To limit this potential bias, we then calculated the lower limit of our institutional PoM by considering as benign all nonresected nodules. Finally, we developed an intermediate PoM, considering as benign all nonresected nodules with at least one sonographic follow-up (i.e. excluding those without follow-up after the biopsy). The estimated NPV and PPV for Afirma, miRInform, and ThyroSeq v2 were calculated based on the sensitivity and specificity reported in extant publications, using Bayes' theorem, whereas 95\% confidence intervals were developed using the Student's $t$-distribution. Specifically, to assess the predicted performance of the Afirma assay (Veracyte Inc., South San Francisco, CA, USA), we used the data published by Alexander and coworkers (8); for miRInform (Asuragen Inc), we used the data published by Beaudenon-Huibregtse and coworkers (9) (sensitivity and specificity for Bethesda III and IV categories were calculated 
Table 1 Baseline demographic and clinical characteristics of the study cohorts.

\begin{tabular}{|c|c|c|c|c|}
\hline & \multicolumn{2}{|c|}{ Cytology cohort } & \multicolumn{2}{|c|}{ miRInform cohort } \\
\hline & All biopsied & Resected & All biopsied & Resected \\
\hline Number of nodules & 2829 & 1103 & 116 & 63 \\
\hline Number of patients & 2175 & 886 & 105 & 56 \\
\hline \multicolumn{5}{|l|}{ Age (years) } \\
\hline Average \pm S.D. & $54 \pm 15$ & $51 \pm 15$ & $56 \pm 14$ & $54 \pm 14$ \\
\hline Range & $8-93$ & $10-86$ & $18-82$ & \\
\hline Female (\%) & $1660(76)$ & $652(74)$ & $86(82)$ & $40(87)$ \\
\hline \multicolumn{5}{|c|}{ Size on ultrasonography $(\mathrm{cm})$} \\
\hline Average \pm S.D. & $2.3 \pm 1.3$ & $2.1 \pm 1.5$ & $2.6 \pm 1.5$ & $2.5 \pm 1.6$ \\
\hline Range & $0.4-10.1$ & $0.4-10$ & $0.9-10$ & $0.9-10$ \\
\hline \multicolumn{5}{|l|}{ Size group } \\
\hline$<1 \mathrm{~cm}(\%)$ & $179(6)$ & $70(6)$ & $5(4)$ & $1(2)$ \\
\hline $1-1.99 \mathrm{~cm}(\%)$ & $1172(41)$ & $413(37)$ & $38(33)$ & $15(24)$ \\
\hline $2-2.99 \mathrm{~cm}(\%)$ & $709(25)$ & $257(23)$ & $41(35)$ & $27(43)$ \\
\hline $3-3.99 \mathrm{~cm}(\%)$ & $358(13)$ & $154(14)$ & $18(16)$ & $9(14)$ \\
\hline$\geq 4 \mathrm{~cm}(\%)$ & $298(11)$ & $155(14)$ & $14(12)$ & $11(17)$ \\
\hline Not available (\%) & $113(44)$ & $54(5)$ & - & - \\
\hline
\end{tabular}

from their Table 2, because they were not provided in the text); and for ThyroSeq v2, we used the data published by Nikiforov and coworkers $(10,11)$

We then reviewed our own institutional experience with miRInform, which was in use between July 2012 and October 2014. Because this was a retrospective study, we recognized that the pathologists reading the histology were aware of both the cytological diagnosis and the oncogene panel result at the time of histological interpretation. Two nodules were tested twice with miRInform during the study period, but, for analysis purposes, each specimen was included only once: no mutation was identified in these two nodules, on either occasion. The actual performance of this test and 95\% confidence intervals were calculated and compared to the predicted performance.

Statistical analysis was performed using SAS version 9.4 (Cary, NC, USA).

\section{Results}

We analyzed a total of 2829 thyroid nodules in 2175 patients (Fig. 1). Sixty-three percent $(n=1777)$ of these nodules were biopsied at our institution, whereas the remaining 37\% ( $n=1052$ ) were biopsied elsewhere but had the cytology slides reviewed by our pathology department. Baseline characteristics of both the patients and the nodules are shown in Table 1. The PoM for each Bethesda category was estimated on 1103 of the 1132 resected nodules after excluding 29 cases (2.6\%) with unclear histological correlation. In these 29 cases, at least one focus of cancer was identified on histological evaluation, but the presence of an adjacent nonmalignant nodule and the lack of information provided in the pathology report did not permit confirmation with confidence that the malignancy represented the biopsied nodule. Surgery was performed at our cancer center for 952 nodules (86\%) and elsewhere in the remaining 151 nodules; of those performed elsewhere, 108 (72\%) histological specimens were available for review by our Pathology department. Overall, our Pathology group issued the histological diagnosis in 1060 (96\%) of 1103 nodules included in the study. The histological diagnosis of the other 43 (4\%) nodules was extracted from outside pathology reports.

The overall PoM in the resected nodules was 50\%. The distribution among Bethesda categories of all the specimens included in the study, as well as the percentage of resection, the percentage of nodules with sonographic follow-up, and the PoM, is shown in Table 2. The rate of resection was 55\% (188/340) in the Bethesda III category and $72 \%(282 / 390)$ in the Bethesda IV category. The PoM within resected nodules (the upper limit of our institutional PoM) was 30\% in Bethesda III specimens and $33 \%$ in Bethesda IV specimens. If we consider "benign" all nonresected thyroid nodules (the lower limit of our institutional PoM), the PoM would be 16 and 23\% for the Bethesda III and IV, respectively. Excluding nodules without ultrasound follow-up after the biopsy (our institutional intermediate PoM), the PoM would be 21 and $28 \%$ for Bethesda III and IV, respectively. The calculated NPV and PPV for the Bethesda III and IV categories for Afirma, miRInform, and ThyroSeq v2, based on these prevalence figures and published sensitivity and specificity data for each test, are shown in Table 3. 
Table 2 Distribution, rates of resection, and follow-up and prevalence of cancer among Bethesda categories.

\begin{tabular}{|c|c|c|c|}
\hline $\begin{array}{l}\text { Bethesda } \\
\text { category }\end{array}$ & $\begin{array}{c}\begin{array}{c}\text { Percentage of } \\
\text { nodules } \\
(n=2829)\end{array} \\
\end{array}$ & $\begin{array}{c}\begin{array}{c}\text { Percentage of } \\
\text { resected (of } \\
\text { category) }(n=1132)\end{array} \\
\end{array}$ & $\begin{array}{c}\text { Percentage } \\
\text { of all } \\
\text { resected }\end{array}$ \\
\hline B-I & $3(95)$ & $34(32)$ & 3 \\
\hline B-II & 55 (1555) & 15 (239) & 21 \\
\hline B-III & $12(340)$ & 55 (188) & 17 \\
\hline B-IV & $14(390)$ & $72(282)$ & 25 \\
\hline$B-V$ & $4(101)$ & $90(91)$ & 8 \\
\hline$B-V I$ & 12 (348) & $86(300)$ & 27 \\
\hline
\end{tabular}

\begin{tabular}{c}
$\begin{array}{c}\text { PoM resected } \\
\text { (highest) } \\
(n=1103), \%\end{array}$ \\
\hline $14(4 / 28)$ \\
$10(24 / 230)$ \\
$30(53 / 178)$ \\
$33(91 / 276)$ \\
$90(82 / 91)$ \\
$>99(299 / 300)$ \\
\hline
\end{tabular}

\begin{tabular}{c}
$\begin{array}{c}\text { Percentage of } \\
\text { US follow-up } \\
(n=904)\end{array}$ \\
\hline $39(37)$ \\
$47(729)$ \\
$23(79)$ \\
$13(50)$ \\
$3(3)$ \\
$2(6)$ \\
\hline
\end{tabular}

\begin{tabular}{ccc}
$\begin{array}{c}\text { PoM follow-up } \\
\text { (intermediate) } \\
(n=2007), \%\end{array}$ & & $\begin{array}{c}\text { PoM all (lowest) } \\
(n=2829), \%\end{array}$ \\
\cline { 1 - 1 } $5(4 / 65)$ & & $4(4 / 95)$ \\
$3(24 / 959)$ & & $2(24 / 1555)$ \\
$21(53 / 257)$ & & $16(53 / 340)$ \\
$28(91 / 326)$ & & $23(91 / 390)$ \\
$87(82 / 94)$ & & $81(82 / 101)$ \\
$99(303 / 307)$ & $94(329 / 348)$ \\
\hline
\end{tabular}

B-I, nondiagnostic; B-II, benign; B-III, atypia/follicular lesion of undetermined significance; B-IV, follicular/Hürthle cell neoplasm; B-V, suspicious for malignancy; B-VI, malignant. PoM, prevalence of malignancy. PoM resected (highest), PoM calculated only on nodules resected in which cytology-histology correlation was clear. PoM follow-up (intermediate), PoM considering benign all nodules not resected with at least one follow-up ultrasound in our records (i.e., excluding patients without follow-up and patients with unclear cytology-histology correlation). In the B-VI category, four additional nodules were counted as malignant: three were intrathyroidal mucosa-associated lymphoid tissue (MALT) lymphomas treated medically, and one was an anaplastic thyroid carcinoma confirmed with a core biopsy. PoM all (lowest), PoM considering benign all nodules not resected (including those without follow-up). In the B-VI category, we have counted 25 additional nodules as malignant as the cytology was diagnostic for anaplastic thyroid carcinoma $(n=3)$ or metastatic deposit of extrathyroidal known malignancy $(n=22)$.

Between July 2012 and October 2014, 116 nodules were tested with miRInform. All cytological and histological specimens in this group were collected at our institution, and the diagnoses were issued by our Anatomic Pathology department in all but two nodules, in a single patient operated elsewhere. Baseline characteristics of these patients and nodules are shown in Table 1. Cytology was indeterminate in $94 \%(n=109)$ of the nodules tested with miRInform (Bethesda III, IV, and V categories). Histological correlation was available in 63 nodules (54\%), and malignancy was confirmed in $41 \%(n=26)$ of the resected nodules. There was partial sample deficit in eight specimens (7\%): RNA failure in six (rearrangements were not evaluated); partial DNA failure in one (RAS mutations were not evaluated); and RNA failure and partial DNA failure in another (rearrangements and KRAS mutations were not evaluated). A mutation was identified in $16 \%$ of the nodules $(n=19)$, and histological correlation was available in $84 \%$ of them $(n=16)$. Benign histology was confirmed in $19 \%$ of resected nodules with a mutation (false positives, $n=3$ ), and a mutation was not identified in $50 \%$ of the cancers in this series (false negatives, $n=13)$. In two nodules, there was partial sample deficit (RNA failure), and therefore, not all of the mutations in the panel could be tested. Correlation between cytology, histology, and molecular results is shown in Table 4 . The general characteristics of the true-positive, false-positive, and false-negative nodules are provided in Table 5. The general performance of the test was calculated for the combined indeterminate group (Bethesda III, IV, and V categories; NPV 74\% and PPV 79\%) and for the Bethesda III (NPV 84\%) and IV (NPV 75\% and PPV 77\%) categories individually (Table 6). The PPV could not be calculated for the Bethesda III category because no mutations were identified in any of these specimens.

\section{Discussion}

This study reports a large thyroid nodule cytologyhistology correlation, using the Bethesda system, to calculate the theoretical performance at our institution of the three most widely used molecular tests. All cytological and almost all histological diagnoses (96\%) included in the study were issued by the Pathology department at our institution, which is important to ensure the internal validation of the results. However, given the retrospective design of the study, patients were managed according to the current standard of care, and therefore, histological confirmation was available in only $40 \%$ of all nodules in this series. Not having the final pathology of $60 \%$ of the nodules is a limitation of this study, but this is driven in particular by observation of most nodules with benign cytology. The rate of resection increased progressively along with the presumed risk of malignancy of each category $(15,55,72,90$, and $86 \%$ for Bethesda II, III, IV, $\mathrm{V}$, and VI specimens, respectively). These rates of resection are similar to, or slightly higher than, those reported in a recent meta-analysis on $>25000$ biopsies (1).

The results of the cytology-histology correlation in this study are similar to other series $(1,2)$, though with several unique characteristics. We observed a higher overall PoM (50\%) compared to the estimated average in a recent meta-analysis (34\%) (1). This difference could be the consequence of several factors: First, many patients with an indeterminate or malignant diagnosis on cytology are referred by their physicians or self-referred to undergo 
Table 3 Theoretical predictive values (and 95\% confidence intervals) of molecular marker tests. Three different values of PoM (presented in bold) were used to calculate the theoretical performance of each test. These PoM are derived from Table 2.

\begin{tabular}{|c|c|c|c|c|c|c|c|c|c|c|}
\hline & \multicolumn{5}{|c|}{ B-III } & \multicolumn{5}{|c|}{ B-IV } \\
\hline & \multirow[t]{2}{*}{ Sn $(\%)$} & \multirow[t]{2}{*}{ Sp (\%) } & \multicolumn{3}{|c|}{ PoM } & \multirow[t]{2}{*}{ Sn (\%) } & \multirow[t]{2}{*}{$\mathrm{Sp}(\%)$} & \multicolumn{3}{|c|}{ PoM } \\
\hline & & & 30 & 21 & 16 & & & 33 & 28 & 23 \\
\hline Afirma & 90 & 53 & & & & 90 & 49 & & & \\
\hline NPV & & & $93(89-96)$ & $95(93-98)$ & $97(95-98)$ & & & $91(87-94)$ & $93(90-95)$ & $94(92-97)$ \\
\hline PPV & & & $45(38-52)$ & $34(28-40)$ & $27(22-31)$ & & & $47(41-52)$ & $41(35-46)$ & $35(30-39)$ \\
\hline miRInform & 36 & 82 & & & & 67 & 92 & & & \\
\hline NPV & & & $75(69-81)$ & $83(78-87)$ & $87(83-91)$ & & & $85(81-89)$ & $88(84-91)$ & $90(87-93)$ \\
\hline PPV & & & $46(39-54)$ & $35(29-41)$ & $28(23-32)$ & & & $80(76-85)$ & $77(72-81)$ & $71(67-76)$ \\
\hline Thyroseq v2 & 91 & 92 & & & & 90 & 93 & & & \\
\hline NPV & & & $96(93-99)$ & $97(96-99)$ & $98(97-100)$ & & & $95(92-98)$ & $96(94-98)$ & 97 (95-99) \\
\hline PPV & & & $83(77-89)$ & $75(62-84)$ & $68(63-73)$ & & & $86(82-90)$ & $83(79-87)$ & $79(75-83)$ \\
\hline
\end{tabular}

B-III, Atypia/Follicular lesion of undetermined significance; B-IV, Follicular/Hürthle cell neoplasm; PoM, prevalence of malignancy; NPV, negative predictive value; PPV, positive predictive value; Sn, sensitivity; Sp, specificity obtained from published data $(9,10,11,12)$.

surgery at our specialized cancer center. Second, many of our patients have another established malignancy, which increases the probability of second primary malignancies $(12,13,14)$. Third, incidental thyroid nodules found during a 18F-fluorodeoxyglucose positron emission tomography scan are a relatively common presentation in our patient population, which also increases the probability of malignancy (15). All of these factors increase the pretest probability of cancer and may limit the generalizability of our data to the broader community. The PoM for our Bethesda V (suspicious-for-malignancy) group (90\%) is one of the highest reported, reflecting the high specificity that our pathologists have for this category. Due to the high PoM observed in this category, no additional benefit from any molecular marker test is expected at our institution, and we have therefore elected not to apply molecular markers to this group of nodules and have excluded them from the analysis of test performance.

We used the mid-point sensitivity and specificity reported in previous clinical validation studies to calculate the theoretical NPV and PPV of the commercially available molecular marker tests on our population. It is important to note that these predictive values are calculated with the estimated sensitivity and specificity of single studies, each of them with limited sample size and wide confidence intervals, which could differ significantly from the true performance. This might be especially relevant in the calculations made for ThyroSeq v2 because the test metrics used for these calculations are derived from a single institution, nonblinded study, whereas for Afirma and miRInform, these parameters are derived from multicenter and blinded studies (8, 9, 10, 11). For example, the original single-center, nonblinded study of the sevengene oncogene panel (upon which miRInform was based) performed rather better than in the more recent multicenter, blinded study, emphasizing the fact that singlecenter results lack external validation $(9,16)$.

Acknowledging the possible limitations of these calculations, theoretically, the highest NPV and PPV should be achieved by ThyroSeq v2 in our cohort. ThyroSeq v2 has been commercialized as a test able to achieve high NPV and PPV in the setting of a broad range of pretest PoM, making it useful for both "rule-in" and "rule-out" cancer for Bethesda III and IV specimens. However, the false-negative rate of ThyroSeq v2 for the Bethesda III specimens may be underestimated because most (84\%) mutation-negative nodules have not been resected in the only published study to date, and therefore, the NPV might be actually lower than reported (11). Afirma, marketed as a "rule-out" test, would also reach the threshold of NPV of $95 \%$ or higher proposed by the NCCN guidelines to favor observation in lieu of surgery on the Bethesda III specimens, if we considered the calculated 'intermediate' or 'lowest' institutional PoM (6). Based on this, Afirma could also reach an NPV close to 95\% in Bethesda IV specimens at our institution. Whether this rather higher residual risk of malignancy justifies the observation in lieu of surgery can only be determined on a case-by-case basis. Finally, miRInform, as a 'rule-in' test, would perform similarly to ThyroSeq v2 in the Bethesda IV category. However, its performance on Bethesda III specimens would be disappointingly low and similar to that achieved by Afirma. Because the original study of the seven-gene oncogene panel in which a posttest PoM of $6 \%$ for Bethesda III mutation-negative specimens was reported (16), observation has been considered acceptable in this subgroup of patients (7). However, our results, in agreement with a more recent, multicenter, blinded 
Table 4 Correlation between cytology, histology, and molecular results. Prevalence of malignancy calculated on resected nodules only.

\begin{tabular}{|c|c|c|c|c|c|}
\hline $\begin{array}{l}\text { Bethesda } \\
\text { category }\end{array}$ & $\begin{array}{c}\text { Number } \\
\text { of } \\
\text { nodules } \\
(n=116)\end{array}$ & $\begin{array}{c}\% \boldsymbol{M}+ \\
(n=19)\end{array}$ & $\begin{array}{c}\text { Number } \\
\text { of } \\
\text { sample } \\
\text { deficits } \\
(n=8)\end{array}$ & $\begin{array}{c}\text { Percentage } \\
\text { of resected } \\
(n, M+) \\
(63,16)\end{array}$ & $\begin{array}{c}\text { Prevalence* } \\
(\%)(n, M+) \\
(26,13)\end{array}$ \\
\hline B-II & 5 & $40(2)$ & 0 & $20(1,1)$ & $100(1,1)$ \\
\hline B-III & 47 & $0(0)$ & 4 & $40(19,0)$ & $16(3,0)$ \\
\hline B-IV & 58 & 26 (15) & 3 & $64(37,13)$ & $43(16,10)$ \\
\hline FN & 40 & 35 (14) & 2 & $63(25,12)$ & $52(13,9)$ \\
\hline $\mathrm{HCN}$ & 18 & $6(1)$ & 1 & $67(12,1)$ & $25(3,1)$ \\
\hline$B-V$ & 4 & $25(1)$ & 1 & $100(4,1)$ & $100(4,1)$ \\
\hline B-VI & 2 & $50(1)$ & 0 & $100(2,1)$ & $100(2,1)$ \\
\hline
\end{tabular}

B-II, benign; B-III, atypia/follicular lesion of undetermined significance; B-IV, follicular/Hürthle cell neoplasm (FN, follicular neoplasm; HCN, Hürthle cell neoplasm); B-V, suspicious for malignancy; B-VI, malignant. $M+$, mutation identified with miRInform.

study using this panel, show a lower NPV that would made observation inappropriate in this setting (9).

Between July 2012 and January 2014, miRInform was used sporadically at our institution. In February 2014, it was included in the standard-of-care work-up for thyroid nodules with indeterminate cytology on our institutional clinical pathway. We present in this report the first independent study published on the performance of this commercially available test. Our observations may be impacted by the fact that nodules with a mutation identified were more likely resected ( $84 \mathrm{vs} 48 \%$ ). Although this strengthens the calculation of the PPV, it may also overestimate the NPV because some false negatives may not yet have been uncovered.

The most striking finding is that none of the 47 samples classified in the Bethesda III category at our institution had a mutation identified by the test, but three cancers were diagnosed in the 19 resected nodules in this group, a false-negative rate of $16 \%$. One of these samples had a partial sample deficit (RNA failure) but proved to be an intrathyroidal parathyroid carcinoma in which none of the mutations studied would be likely to occur. The other two cancers were a follicular thyroid carcinoma (FTC) and a follicular variant of papillary thyroid carcinoma (FVPTC), without any mutation or rearrangement identified, despite confirmed sample integrity. The rate of mutations in Bethesda III specimens was lower than in the Bethesda IV category in single-center publications using either the seven-gene oncogene panel or the more sensitive oncogene panel ThyroSeq $\mathrm{v} 2$, in part due to the lower PoM of this category $(11,16)$. In fact, in the most recent publication using ThyroSeq v2, a mutation was identified in less than $7 \%$ of the 465 nodules tested with a Bethesda III diagnosis (11). However, the PoM of both categories at our institution is similar (30 and 33\% for resected nodules), consistent with the heterogeneity of this cytological category in reported studies (2). This heterogeneity would be expected to impact not only the PoM but also the histological findings at the time of surgery probably causing significant differences on test performance among institutions. If comparable performance of these tests is desired among centers, the inclusion and exclusion criteria of the Bethesda III category need to be better defined and probably need to be subcategorized to improve risk stratification $(17,18,19)$.

In our study, a single mutation was detected among the 18 nodules with cytologic diagnosis of Hürthle cell neoplasm (HCN). In most publications, these nodules are analyzed together with the follicular neoplasms, because they are grouped under the same Bethesda category. However, in our opinion, this differentiation is fundamental for the application of molecular markers. It is known that the most common mutations present in thyroid cancer (evaluated by miRInform) are not usually present in Hürthle cell carcinoma (HCC) (20). In the initial report of ThyroSeq, a mutation was identified in $39 \%$ of the HCCs (21), suggesting that the broader panel might detect some of these malignancies. However, this finding needs further independent and prospective validation, and these results probably would be insufficient to avoid surgery if a mutation is not identified. However, despite having a lower risk of malignancy than other follicular neoplasms, $65-90 \%$ of HCNs are classified as suspicious by Afirma $(22,23)$. Although the NPV in benign HCN by Afirma seems to be preserved, the cost-effectiveness in this setting is significantly challenged.

It is notable that we tested five nodules with benign cytology and found a mutation in two of them (NRAS Q61K in both). Both had a prior biopsy read as indeterminate that had prompted the repeat biopsy with sample collection for the oncogene panel. One patient had surgery that confirmed an encapsulated, noninvasive FVPTC. The other is being followed, and the nodule has not grown significantly after a follow-up of $>2$ years. One of the other three nodules with benign cytology, without an identified mutation, also had a prior indeterminate cytology, whereas in the remaining two nodules, it was unclear why the test was performed. We also found a surprisingly low rate of mutations in the suspicious-formalignancy and diagnostic-for-malignancy categories, albeit with only small numbers tested $(2 / 6,33 \%)$. Interestingly, one of the nodules classified as malignant by the 
Table 5 Characteristics of "true-positive," "false-positive," and "false-negative" nodules.

\begin{tabular}{l} 
Number \\
\hline True positives $(n=13)$ \\
1 \\
2 \\
3 \\
4 \\
5 \\
6 \\
7 \\
8 \\
9 \\
10 \\
11 \\
12 \\
13 \\
False positives $(n=3)$ \\
1 \\
2 \\
3 \\
Unresected $(n=3)$ \\
1 \\
2 \\
3 \\
False negatives $(n=13)$ \\
1 \\
2 \\
3 \\
4 \\
5 \\
6 \\
7 \\
8 \\
9 \\
10 \\
11 \\
12 \\
13 \\
\end{tabular}

\begin{tabular}{l} 
Mutation \\
\hline HRAS (Q61R) \\
KRAS (G12V) \\
NRAS (Q61K) \\
NRAS (Q61K) \\
NRAS (Q61R) \\
NRAS (Q61R) \\
NRAS (Q61R) \\
NRAS (Q61R) \\
NRAS (Q61R) \\
NRAS (Q61R) \\
NRAS (Q61R)+RETIPTC1 \\
PAX8/PPARG \\
PAX8IPPARG \\
KRAS (G12C) \\
NRAS (Q61K) \\
NRAS (Q61R) \\
NRAS (Q61K) \\
NRAS (Q61R) \\
NRAS (Q61R) \\
- \\
- \\
- \\
- \\
- \\
- \\
- \\
- \\
- \\
- \\
- \\
- \\
\hline
\end{tabular}

Age

Size $(\mathrm{cm})$

Bethesda category

Histology

$\begin{array}{lll}60 & 2.6 & \text { F/HCN } \\ 45 & 3.8 & \text { Malignant } \\ 71 & 2.8 & \text { Benign (prior F/HCN) } \\ 48 & 2.8 & \text { F/HCN } \\ 57 & 3.1 & \text { F/HCN } \\ 25 & 1.5 & \text { F/HCN }\end{array}$

FVPTC

$32 \quad 3.5 \quad \mathrm{~F} / \mathrm{HCN}$

$335.2 \quad \mathrm{~F} / \mathrm{HCN}$

FTC

FVPTC

FVPTC

FTC

FVPTC

FVPTC

FVPTC

FVPTC

FVPTC

FVPTC

FVPTC

FVPTC

$56 \quad 3.3$

\section{F/HCN}

HCA

49

2.4
2.6

F/HCN

FA

Ad. nodule

55

46

84

1.9

2.8

1.8

Benign (prior A/FLUS)

F/HCN

F/HCN

A/FLUS

$\begin{array}{ll}3.8 & \text { A/FLUS } \\ 1.4 & \text { A/FLUS }\end{array}$

0.2 F/HCN

$7 \quad \mathrm{~F} / \mathrm{HCN}$

$1.8 \quad \mathrm{~F} / \mathrm{HCN}$

$0.9 \quad \mathrm{~F} / \mathrm{HCN}$

$1 \quad \mathrm{~F} / \mathrm{HCN}$

1 F/HCN

5.5 SM

2.2 SM

1.7 SM

1.2

A/FLUS, atypia/follicular lesion of undetermined significance; F/HCN, follicular/Hürthle cell neoplasm; SM, suspicious for malignancy; Ad. nodule, adenomatous nodule; FA, follicular adenoma; HCA, Hürthle cell adenoma; FTC, follicular thyroid carcinoma; FVPTC, follicular variant of papillary thyroid carcinoma; HCC, Hürthle cell carcinoma; PTH-Ca, parathyroid carcinoma; OVPTC, oncocytic variant of papillary thyroid carcinoma.

aSample deficit (RNA failure); ${ }^{\text {b } 2 ~ m m ~ P T C ~ a r i s i n g ~ w i t h i n ~ t h e ~} 1.5$ biopsied nodule that was considered an adenomatous nodule.

cytology (called PTC on cytology) was actually classified as FTC on histology. The cytology was reviewed after the histological diagnosis and the classification remained the same, because there were numerous oncocytic cells with prominent nucleoli, grooves, and rare pseudoinclusions identified, which made it difficult to rule out a PTC on smears alone. However, cells on the cytological specimens were identical to the FTC cells noted on the resection specimen, which was best classified as an encapsulated, minimally invasive FTC. The other five tumors were FVPTC, one of which had a mutation identified.

Despite being the most common mutation among thyroid cancers, no BRAF-V600E (BRAF) mutations were detected in this series. This is in part because most speci- mens submitted for molecular testing were classified as Bethesda III and IV, in which the most prevalent cancers are the FTC and the FVPTC. In fact, they represent $88.5 \%$ of the cancers in our series ( $n=4$ and $n=19$, respectively). $B R A F$ is not present in FTC and infrequent in the FVPTC (around 25\% exhibit a BRAF mutation), and therefore, $B R A F$ is a mutation rarely found in thyroid nodules with indeterminate cytology $(24,25)$. This also reflects the sensitivity that our pathologists have for the typical nuclear features of PTC, which are more frequently present in tumors with a mutated $B R A F$, and therefore classified as malignant by cytology (26). In our series, $58 \%$ of the FVPTC $(n=11)$ had a PAX/PPARG rearrangement or a RAS mutation (one of them in combination with a RET/PTC1 
Table 6 Actual performance of miRInform for the indeterminate categories at Moffitt with 95\% confidence intervals.

\begin{tabular}{|c|c|c|c|c|c|c|c|}
\hline Categories & Malignant & Benign & Sensitivity & Specificity & NPV & PPV & Accuracy \\
\hline B-III & & & $0 \%(0-69)$ & $100 \%(76-100)$ & $84 \%(60-97)$ & - & - \\
\hline Total $(n=19)$ & 3 & 16 & & & & & \\
\hline $\mathrm{M}+$ & 0 & 0 & & & & & \\
\hline M- & 3 & 16 & & & & & \\
\hline B-IV & & & $62.5 \%(36-84)$ & $85.7 \%(63-96)$ & $75 \%(53-90)$ & $77 \%(46-95)$ & $76 \%(59-88)$ \\
\hline Total $(n=37)$ & 16 & 21 & & & & & \\
\hline $\mathrm{M}+$ & 10 & 3 & & & & & \\
\hline M- & 6 & 18 & & & & & \\
\hline B-III, IV and V & & & $48 \%(27-69)$ & $92 \%(78-98)$ & $74 \%(59-86)$ & $79 \%(49-95)$ & $75 \%(62-85)$ \\
\hline Total $(n=60)$ & 23 & 37 & & & & & \\
\hline $\mathrm{M}+$ & 11 & 3 & & & & & \\
\hline M- & 12 & 34 & & & & & \\
\hline
\end{tabular}

B-III, Atypia/Follicular lesion of undetermined significance; B-IV, Follicular/Hürthle cell neoplasm; B-V, Suspicious for malignancy; M+, Mutation positive; M-, Mutation negative.

rearrangement), whereas no mutation was detected in the other $42 \%(n=8)$. A RAS mutation was also found in two FTC and three benign nodules (one follicular adenoma, one Hürthle cell adenoma, and one adenomatoid nodule). Three nodules with a RAS mutation have not yet been resected. Concordant with prior reports, 79\% (11/14) of the resected nodules with a $R A S$ mutation proved to be malignant on final histology, most of them being low-risk follicular pattern cancers $(9,16,27,28)$.

In this study, we determined our institutional average PoM for each cytological category to optimize the use and interpretation of molecular marker tests. However, this is merely the first step toward an accurate interpretation, because the PoM for a given cytological category can only be accurately measured if all nodules in that category are resected. Moreover, the PoM may change over time in response to practice changes and availability of molecular marker testing. It is also critical to recognize that the NPV and PPV should really be individualized for each nodule as other factors such as the sonographic appearance or the cytological features might also impact the pretest PoM $(17,18,19)$. Although our results with the seven-gene oncogene panel might not be applicable to other institutions, miRInform performed better on follicular neoplasms than on HCN or Bethesda III samples, for which it might have limited utility.

Despite all the study limitations described previously, our results remain relevant, because they highlight the need to tailor the use of these molecular marker tests to the outcomes of each institution's cytology reporting and not only to the average PoM. In our opinion, studies like this should be conducted in any center where molecular markers are being considered for use, or where these tests are already part of routine clinical practice to guide clinicians on the selection of the most appropriate test and the interpretation of the results. Until those data are available, molecular markers should be used with caution, although patients who avoid surgery on the basis of negative molecular testing should be offered more careful and more prolonged surveillance than would be typical for patients with truly benign cytology.

\section{Declaration of interest}

Dr Mclver reports receiving payments for scientific consulting services from Veracyte, South San Francisco, CA, USA, Asuragen, Austin, TX, USA, and CBLPath, Rye Brook, NY, USA (manufacturers or sellers of Afirma, miRInform, and Thyroseq V2, respectively) between 2011 and 2014. No other conflicts of interest exist. Dr Leon has received sponsored research funding from Rosetta Genomics. The remaining authors declare that there is no conflict of interest that could be perceived as prejudicing the impartiality of the research reported.

\section{Funding}

This research did not receive any specific grant from any funding agency in the public, commercial, or not-for-profit sector.

\section{Acknowledgments}

Pablo Valderrabano gratefully acknowledges the financial support from the Alfonso Martín Escudero Foundation (Spain).

\section{References}

1 Bongiovanni M, Spitale A, Faquin WC, Mazzucchelli L \& Baloch ZW. The Bethesda System for Reporting Thyroid Cytopathology: a metaanalysis. Acta Cytologica 201256 333-339. (doi:10.1159/000339959)

2 Wang CC, Friedman L, Kennedy GC, Wang H, Kebebew E,

Steward DL, Zeiger MA, Westra WH, Wang Y, Khanafshar E, et al. A large multicenter correlation study of thyroid nodule cytopathology and histopathology. Thyroid 201121 243-251. (doi:10.1089/ thy.2010.0243)

3 Bergenfelz A, Jansson S, Kristoffersson A, Martensson H, Reihner E, Wallin G \& Lausen I. Complications to thyroid surgery: results 
as reported in a database from a multicenter audit comprising 3,660 patients. Langenbeck's Archives of Surgery 2008393 667-673. (doi:10.1007/s00423-008-0366-7)

4 Zevallos JP, Hartman CM, Kramer JR, Sturgis EM \& Chiao EY. Increased thyroid cancer incidence corresponds to increased use of thyroid ultrasound and fine-needle aspiration: a study of the Veterans Affairs health care system. Cancer 2015121 741-746. (doi:10.1002/ cncr.29122)

5 Haugen BRM, Alexander EK, Bible KC, Doherty G, Mandel SJ, Nikiforov YE, Pacini F, Randolph G, Sawka A, Schlumberger M, et al. American Thyroid Association Management Guidelines for adult patients with thyroid nodules and differentiated thyroid cancer. Thyroid 201626 1-133. (doi:10.1089/thy.2015.0020)

6 National Comprehensive Cancer Network. NCCN Clinical Practice Guidelines in Oncology (Thyroid Carcinoma). Version I. 2015. 2015. (doi:10.5603/EP.2016.0011)

7 Ferris RL, Baloch ZW, Bernet V, Chen A, Fahey T, 3rd, Ganly I, Hodak S, Kebebew E, Patel KN, Shaha ARM, et al. American Thyroid Association Statement on Surgical Application of Molecular Profiling for Thyroid Nodules: current impact on perioperative decisionmaking. Thyroid 201525 760-768. (doi:10.1089/thy.2014.0502)

8 Alexander EK, Kennedy GC, Baloch ZW, Cibas ES, Chudova D, Diggans J, Friedman L, Kloos RT, LiVolsi VA, Mandel SJ, et al. Preoperative diagnosis of benign thyroid nodules with indeterminate cytology. New England Journal of Medicine 2012367 705-715. (doi:10.1056/NEJMoa1203208)

9 Beaudenon-Huibregtse S, Alexander EK, Guttler RB, Hershman JM, Babu V, Blevins TC, Moore P, Andruss B \& Labourier E. Centralized molecular testing for oncogenic gene mutations complements the local cytopathologic diagnosis of thyroid nodules. Thyroid $2014 \mathbf{2 4}$ 1479-1487. (doi:10.1089/thy.2013.0640)

10 Nikiforov YE, Carty SE, Chiosea SI, Coyne C, Duvvuri U, Ferris RL, Gooding WE, Hodak SP, LeBeau SO, Ohori NP, et al. Highly accurate diagnosis of cancer in thyroid nodules with follicular neoplasm/ suspicious for a follicular neoplasm cytology by ThyroSeq v2 next-generation sequencing assay. Cancer 2014120 3627-3634. (doi:10.1002/cncr.29038)

11 Nikiforov YE, Carty SE, Chiosea SI, Coyne C, Duvvuri U, Ferris RL, Gooding WE, LeBeau SO, Ohori NP, Seethala RR, et al. Impact of the multi-gene thyroseq next-generation sequencing assay on cancer diagnosis in thyroid nodules with atypia of undetermined significance/follicular lesion of undetermined significance cytology. Thyroid 201525 1217-1223. (doi:10.1089/thy.2015.0305)

12 Lal G, Groff M, Howe JR, Weigel RJ, Sugg SL \& Lynch CF. Risk of subsequent primary thyroid cancer after another malignancy: latency trends in a population-based study. Annals of Surgical Oncology 2012 19 1887-1896. (doi:10.1245/s10434-011-2193-2)

13 Morton LM, Onel K, Curtis RE, Hungate EA \& Armstrong GT. The rising incidence of second cancers: patterns of occurrence and identification of risk factors for children and adults. American Society of Clinical Oncology Educational Book 2014 e57-e67.

14 Van Fossen VL, Wilhelm SM, Eaton JL \& McHenry CR. Association of thyroid, breast and renal cell cancer: a population-based study of the prevalence of second malignancies. Annals of Surgical Oncology2013 20 1341-1347. (doi:10.1245/s10434-012-2718-3)

15 Soelberg KK, Bonnema SJ, Brix TH \& Hegedus L. Risk of malignancy in thyroid incidentalomas detected by $18 \mathrm{~F}$-fluorodeoxyglucose positron emission tomography: a systematic review. Thyroid 201222 918-925. (doi:10.1089/thy.2013.0136)

16 Nikiforov YE, Ohori NP, Hodak SP, Carty SE, LeBeau SO, Ferris RL, Yip L, Seethala RR, Tublin ME, Stang MT, et al. Impact of mutational testing on the diagnosis and management of patients with cytologically indeterminate thyroid nodules: a prospective analysis of 1056 FNA samples. Journal of Clinical Endocrinology and Metabolism 201196 3390-3397. (doi:10.1210/jc.2011-1469)

17 Kato MA, Buitrago D, Moo TA, Keutgen XM, Hoda RS, Ricci JA, Christos PJ, Yang G, Fahey TJ, III \& Zarnegar R. Predictive value of cytologic atypia in indeterminate thyroid fine-needle aspirate biopsies. Annals of Surgical Oncology 201118 2893-2898. (doi:10.1245/s10434-011-1635-1)

18 Mathur A, Najafian A, Schneider EB, Zeiger MA \& Olson MT. Malignancy risk and reproducibility associated with atypia of undetermined significance on thyroid cytology. Surgery 2014156 1471-1476. (doi:10.1016/j.surg.2014.08.026)

19 Rosario PW. Thyroid nodules with atypia or follicular lesions of undetermined significance (Bethesda Category III): importance of ultrasonography and cytological subcategory. Thyroid $2014 \mathbf{2 4}$ 1115-1120. (doi:10.1089/thy.2013.0650)

20 Filicori F, Keutgen XM, Buitrago D, AlDailami H, Crowley M, Fahey TJ, 3rd \& Zarnegar R. Risk stratification of indeterminate thyroid fine-needle aspiration biopsy specimens based on mutation analysis. Surgery 2011150 1085-1091.

21 Nikiforova MN, Wald AI, Roy S, Durso MB \& Nikiforov YE. Targeted next-generation sequencing panel (ThyroSeq) for detection of mutations in thyroid cancer. Journal of Clinical Endocrinology and Metabolism 201398 E1852-E1860. (doi:10.1210/ jc.2013-2292)

22 Harrell RM \& Bimston DN. Surgical utility of afirma: effects of high cancer prevalence and oncocytic cell types in patients with indeterminate thyroid cytology. Endocrine Practice 201420 364-369. (doi:10.1016/j.amjmed.2015.04.044)

23 Brauner E, Holmes BJ, Krane JF, Nishino M, Zurakowski D, Hennessey JV, Faquin WC \& Parangi S. Performance of the afirma gene expression classifier in hurthle cell thyroid nodules differs from other indeterminate thyroid nodules. Thyroid 201525 789-796. (doi:10.1089/thy.2015.0049)

24 Li C, Lee KC, Schneider EB \& Zeiger MA. BRAF V600E mutation and its association with clinicopathological features of papillary thyroid cancer: a meta-analysis. Journal of Clinical Endocrinology and Metabolism 201297 4559-4570. (doi:10.1210/jc.2013-1584)

25 Trimboli P, Treglia G, Condorelli E, Romanelli F, Crescenzi A, Bongiovanni M \& Giovanella L. BRAF-mutated carcinomas among thyroid nodules with prior indeterminate FNA report: a systematic review and meta-analysis. Clinical Endocrinology 201684 315-320. (doi:10.1111/cen.2016.84.issue-3)

26 Kleiman DA, Sporn MJ, Beninato T, Crowley MJ, Nguyen A, Uccelli A, Scognamiglio T, Zarnegar R \& Fahey TJ, 3rd. Preoperative $\mathrm{BRAF}(\mathrm{V} 600 \mathrm{E})$ mutation screening is unlikely to alter initial surgical treatment of patients with indeterminate thyroid nodules: a prospective case series of 960 patients. Cancer 2013119 1495-1502. (doi:10.1002/cncr.27888)

27 Gupta N, Dasyam AK, Carty SE, Nikiforova MN, Ohori NP, Armstrong M, Yip L, LeBeau SO, McCoy KL, Coyne C, et al. RAS mutations in thyroid FNA specimens are highly predictive of predominantly low-risk follicular-pattern cancers. Journal of Clinical Endocrinology and Metabolism 201398 E914-E922. (doi:10.1210/ jc.2012-3396)

28 Radkay LA, Chiosea SI, Seethala RR, Hodak SP, LeBeau SO, Yip L, McCoy KL, Carty SE, Schoedel KE, Nikiforova MN, et al. Thyroid nodules with KRAS mutations are different from nodules with NRAS and HRAS mutations with regard to cytopathologic and histopathologic outcome characteristics. Cancer Cytopathology 2014 122 873-882. (doi:10.1002/cncy.21474)

Received 25 November 2015

Revised version received 1 February 2016

Accepted 17 February 2016 
Downloaded from Bioscientifica.com at 04/26/2023 12:25:27PM

via free access 